International Journal of Advancement in Life Sciences Research

Online ISSN: 2581-4877

journal homepage http://ijalsr.org

Original Article

\title{
Retrospective Analysis of Antibiotic Prescribing Pattern for Upper Respiratory Tract Infection (URTI) in Seremban 2 Health Clinic
}

\author{
Chong Zhi Juan, Melissa Soon Pei Shan* \\ Pharmacy Department, Seremban 2 Health Clinic, Seremban Health District Office, Negeri Sembilan \\ State Health Department, Malaysia
}

CorrespondenceE-mail : zhijuanchong@gmail.com ; melissa_sps@yahoo.com*

\begin{abstract}
Introduction: URTI is a common problem seen in primary care. URTIs are often of viral origin and therefore the use of antibiotics are not necessary. Overprescribing of antibiotics can lead to antibiotic resistance.

Objectives: 1) Determine the antibiotic prescribing pattern for URTI at Seremban 2 Health Clinic. 2) Determine the appropriateness of antibiotics prescribed for URTI based on Malaysian National Antibiotic Guideline (MNAG) 2014 and the defined daily dose (DDD) system introduced by WHO.

Method: Information was collected from Pharmacy Information System (PhIS). All records of patients attending the clinic from $1^{\text {st }}$ January 2018 to $31^{\text {st }}$ December 2018 , who fulfilled the necessary criteria were screened and retrieved for further investigation. The data obtained was analysed using IBM SPSS Statistics version 25.0 (IBM Corp., Armonk, NY, USA).

Results: URTI was found to be the most common diagnosis, which accounted for about $27.0 \%$ from the total diagnosis for which antibiotics were prescribed. The overall antibiotic prescribing rate and URTI antibiotic prescribing rate in the current study were $11.6 \%$ and $25.2 \%$ respectively. Amoxicillin $(49.8 \%)$ was the most frequently prescribed antibiotic for the indication of URTI followed by erythromycin $(43.4 \%)$, cloxacillin $(2.8 \%)$, amoxicillin/clavulanic acid $(1.8 \%)$, cephalexin $(1.8 \%)$, cefuroxime $(0.3 \%)$ and doxycycline $(0.2 \%)$. The most utilized antibiotics for URTI was amoxicillin $(1.31$ DDD/100 patients), and was used almost 3-fold more than erythromycin which was the second most utilized antibiotic for URTI (0.48 DDD/100 patients).
\end{abstract}

Conclusion: These results show that the current practice is not following the antibiotic guidelines.

Keywords: antibiotic prescribing pattern, upper respiratory tract infection, Malaysia.

\section{Introduction}

Upper respiratory tract infection (URTI) is a common problem seen in primary care. In 2003, studies carried out in Malaysia on antibiotic prescribing rates for URTI at primary healthcare settings have revealed an overall antibiotic prescribing rate of $28.7 \%$ (Teng et al. 2003). Another study was carried out years later in 2011 at primary healthcare settings in Seremban and the antibiotic prescribing rate was reported to be $33.8 \%$ for URTI (Cheong et al. 2011). URTIs are often of viral origin and therefore the use of antibiotics are not necessary (Fikru and Dagmawit 2018). Hence, overprescribing of antibiotics can lead to 
antibiotic resistance (Zeyad et al. 2017). Antibiotic resistance has been a long-existing universal health threat. 700,000 people worldwide die per annum due to drug-resistant infections, and this will rise to 10 million deaths per annum by the year 2050 which was proposed by a scenario analysis, if no international action is taken (O'Neill J. 2016). According to World Health Organization (WHO 2018), antibiotic resistance is a burgeoning and often neglected issue in Southeast Asia. It is believed that a 'post-antibiotic era' may come true, causing failure in treatments of minor injuries and common infections. Following an antibiotic course, antibiotic resistance is evident for one month and can last for up to 12 months in an individual patient (Costelloe et al. 2010). Deviation from guideline-recommended practice is often seen when it comes to dealing with respiratory problems (Rezel et al. 2015), tonsillitis (RicoFerreira et al.2015), acute diarrhoea (Boonztra et al. 2005), and urinary tract infections Philips et al. 2014). Strong evidence has supported prescribing the smallest amount of antibiotic courses for the shortest possible period of time (O'Neill J. 2016). This emergence is contributed by indiscriminate antibiotic prescribing, which includes inappropriate and irrational prescribing of antibiotics in healthcare settings (Cheong et al. 2011). This will eventually lead to treatment failure, poor clinical outcomes, death of patients and other problems such as wastage of healthcare resources and non-adherence issues (O'Neill J. 2016). Among the factors contributing to inappropriate prescribing of antibiotics include inadequate experience and knowledge of prescriber, parental or patient pressure and individual preferences (Akter S.F. 2012). Although guidelines have been provided by the Malaysian Ministry of Health $(\mathrm{MoH})$ as a basis to ensure the appropriate use of antibiotics in the healthcare setting, it is impossible to guarantee that these guidelines are meticulously followed (Shafinaz et al. 2016). Thus, prescribers and patients both play a vital role in ensuring appropriate use of antibiotics. Information on the appropriateness of antibiotics prescribed among adults in the primary healthcare setting is important to determine the effectiveness of the antibiotics prescribed. $\mathrm{WHO}(2018)$ has introduced a technical unit of measurement for drug usage called the defined daily dose (DDD). DDD is the assumed average maintenance dose per day for a drug used for its main indication in adults. DDD does not necessarily reflect the recommended or actual dose used. DDD allows international comparisons and study changes in utilization of drug over time. In this context, we felt that it is necessary to know the consumption of antibiotics in Seremban 2 Health Clinic as this can be an important indicator of the quality and standard of clinical practice among prescribers in the clinic. Therefore, a study was conducted to analyse the antibiotic prescribing pattern for URTI at Seremban 2 Health Clinic in Seremban, Negeri Sembilan, Malaysia.

\section{Objectives}

1. Determine the antibiotic prescribing pattern for URTI at Seremban 2 Health Clinic.

2. Determine the appropriateness of antibiotics prescribed for URTI based on Malaysian National Antibiotic Guideline (MNAG) 2014 and the defined daily dose (DDD) system introduced by WHO.

\section{Materials and Methods}

The study was conducted in Seremban 2 Health Clinic in Seremban, Negeri Sembilan, Malaysia. The clinic is fully funded by the government and under the management of Seremban Health District Office. There are a total of 2835 public health clinics scattered throughout Malaysia (MOH 2018). Seremban is the capital town of Negeri Sembilan and has a population of 372,917 (WPR 2018). Services provided at this clinic include outpatient, pharmacy, maternal and child health, dental, health education and promotion, smoking cessation, methadone, laboratory, radiology and rehabilitation services. Patients attending the clinic are seen by medical doctors and family medicine specialists.

Information was collected from Pharmacy Information System (PhIS). All records of patients attending the clinic from $1^{\text {st }}$ January 2018 to $31^{\text {st }}$ December 2018, who fulfilled the 
necessary criteria were screened and retrieved for further investigation. Inclusion criteria comprise of patients aged between 18 and 60 years old and given oral antibiotics who attended the clinic from $1^{\text {st }}$ January 2018 to $31^{\text {st }}$ December 2018. The exclusion criteria are patients who were given antibiotics in injection form, syrup form or topical antibiotics as well as dental patients, Sistem Pendispensan Ubat Bersepadu (SPUB) patients and walk-in patients.

The appropriateness of antibiotics prescribed for URTI was evaluated by referring to MNAG 2014 and the DDD system introduced by WHO. DDD analysis was carried out by comparing the DDD results with the current WHO classification of ATC/DDD (WHO 2018). In this study, the data is presented as DDD per 100 patients per day. The quantity of drug used was calculated as follow (Shatinaz et al. 2016):

$$
\frac{D D D}{100 \text { patients perday }}=\mathrm{T} \times \frac{100}{D D D \times P}
$$

Where:

$\mathrm{T}=$ the total quantity of the drug in gm utilized

DDD = DDD assigned for the drug according to the ATC/DDD system

$\mathrm{P}=$ total number of patients who attended the pharmacy

The DDD was compared to the treatment agreed in the MNAG to assess the prescribing quality and appropriateness.

Electronic prescriptions with diagnosis of URTI, including tonsillitis, pharyngitis, laryngitis, sinusitis, rhinitis and otitis media were included in the study. Cough and sore throat were also included as separate diagnosis as they are commonly encountered respiratory complaints at healthcare settings (Rabiatul et al. 2015). The appropriateness of the antibiotics prescribed was reviewed based on MNAG 2014. The prescribed medicine, dose, and frequency were recorded for each patient to calculate the DDD per 100 patients per day.

For this study, 95\% confidence level was chosen, which is equal to Z-score of 1.96 . Standard deviation was set at 0.5 , and a margin error of $+/-5 \%$. The sample size calculated for this study was 385 respondents. Data recorded in data collection forms were transferred to Microsoft Excel version 2013. The records will provide information regarding patient demographic data, diagnoses, types of antibiotics prescribed and antibiotic regimen. The data obtained was analysed using IBM SPSS Statistics version 25.0 (IBM Corp., Armonk, NY, USA). Statistical significance was set at $p<0.05$. Descriptive statistics such as the mean and standard deviation (SD) were used for data elaboration.

\section{Results}

A total of 24,488 patients attended the pharmacy at Seremban 2 Health Clinic between January and December 2018. 3865 patients were prescribed with at least one antibiotic throughout the year. Among the 35,397 prescriptions dispensed, 4120 (11.6\%) prescriptions contained antibiotics. All antibiotic prescriptions were screened based on the inclusion and exclusion criteria. URTI was found to be the most common diagnosis, which accounted for about $27.0 \%$ from the total diagnosis for which antibiotics were prescribed. A total of $2588(62.7 \%)$ antibiotic prescriptions fulfilled the inclusion criteria, of which 651 (25.2\%) prescriptions indicated diagnosis for URTI and were eligible for further analysis and investigation.

\section{Diagnosis for indication of URTI}

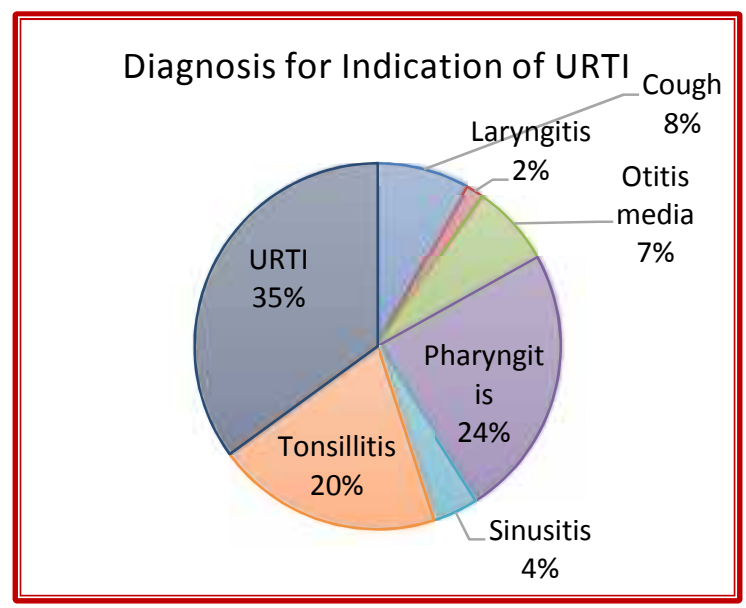

Figure 1: Pie chart showing diagnosis written by prescribers for indication of URTI

Findings showed that antibiotics were often prescribed for the diagnosis of URTI (35\%), pharyngitis (24\%) and tonsillitis (20\%). 


\section{Antibiotic Prescribing Pattern}

A total of 7 antibiotics were prescribed for the indication of URTI, namely amoxicillin, amoxicillin/clavulanic acid, cefuroxime, cloxacillin, doxycycline, erythromycin and cephalexin. Figure 2 shows amoxicillin
(49.8\%) being the most frequently prescribed antibiotic for the indication of URTI followed by erythromycin (43.4\%), cloxacillin $(2.8 \%)$, amoxicillin/clavulanic acid (1.8\%), cephalexin $(1.8 \%)$, cefuroxime $(0.3 \%)$ and doxycycline $(0.2 \%)$.

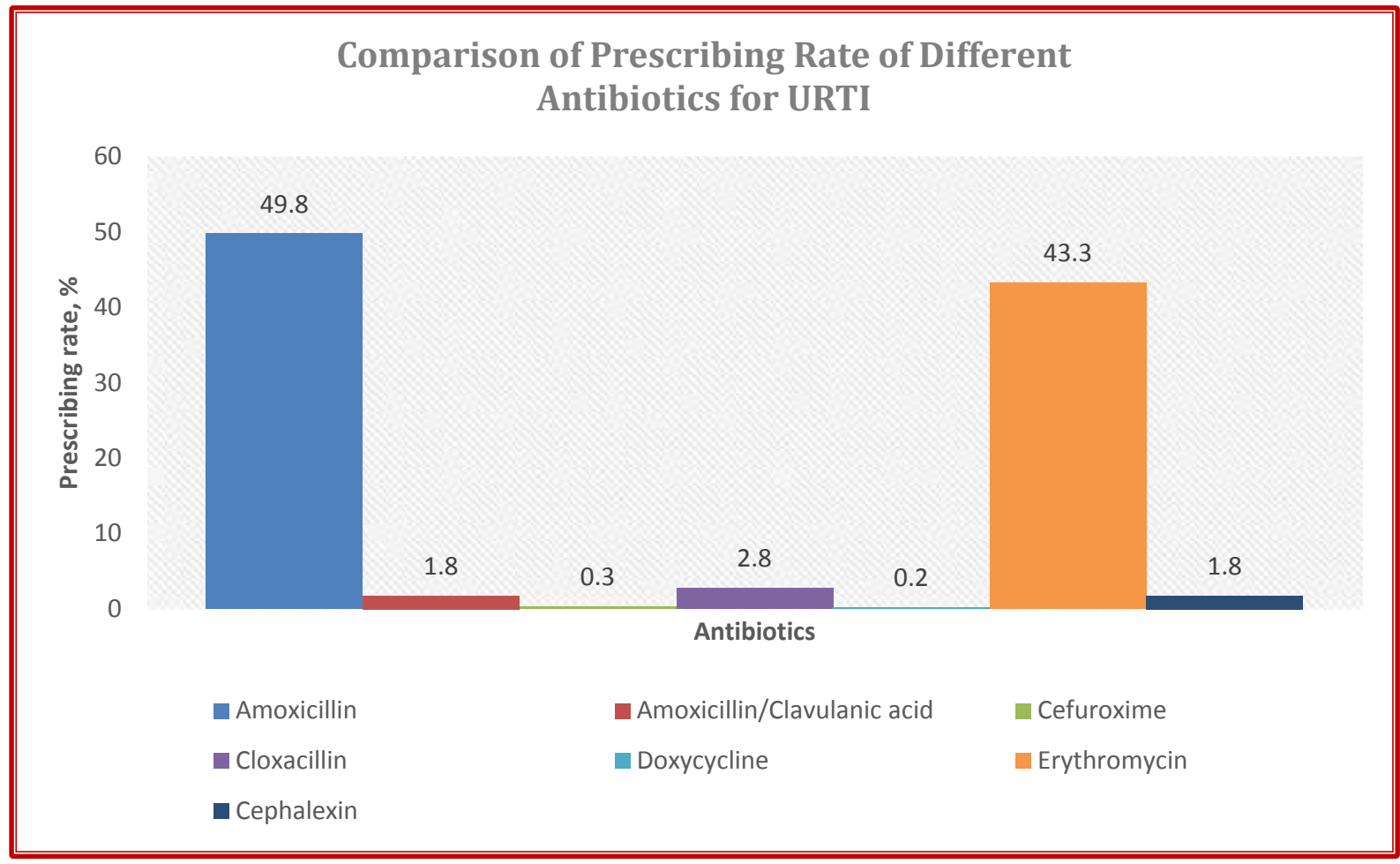

Figure 2: Comparison of antibiotic prescribing rate for the indication of URTI

\section{Socio-demographic characteristics}

Table 1: Demographic characteristics of patients prescribed with antibiotics for URTI

\begin{tabular}{|l|l|}
\hline Gender & $(\%)$ \\
Male & $286(43.9)$ \\
\hline Female & $365(56.1)$ \\
\hline Subtotal & $651(100)$ \\
\hline & \\
\hline Ethnicity & $(\%)$ \\
\hline Malay & $414(63.6)$ \\
Chinese & $74(11.4)$ \\
\hline Indian & $145(22.3)$ \\
\hline Others & $18(2.8)$ \\
\hline Subtotal & $651(100)$ \\
\hline & \\
\hline Age Group (years) & $(\%)$ \\
\hline $18-29$ & $241(37)$ \\
\hline $30-39$ & $182(27.9)$ \\
$40-49$ & $126(19.4)$ \\
\hline $50-60$ & $102(15.7)$ \\
Subtotal & $651(100)$ \\
\hline
\end{tabular}

A total of 651 patients were analysed. As shown in Table 1, there were more females than males $(56.1 \%$ vs. $43.9 \%)$. Malays contributed to more than half of the study population $(63.6 \%)$. This is followed by Indians (22.3\%), Chinese $(11.4 \%)$ and other ethnicities (2.8\%) (Table 1). The mean age of the patients was $35.22( \pm 11.67)$ years. Referring to Table 1, patients aged 18-29 years old contributed slightly more than one third of the total study population whereas patients aged 50-60 years old only made up of less than one fifth of the study population. Patients' gender and age groups were found not to have a statistically significant association with antibiotic prescribing ( $p>0.05)$.

\section{Factors affecting prescribing pattern according to gender}

Overall, the prescribing rate of antibiotics for females were higher for all the antibiotics except for cefuroxime and cloxacillin, where 
the prescribing rate was the same between females and males (Figure 3). The prescribing rate of antibiotics for females do not have a statistically significant difference from males ( $p>0.05)$.

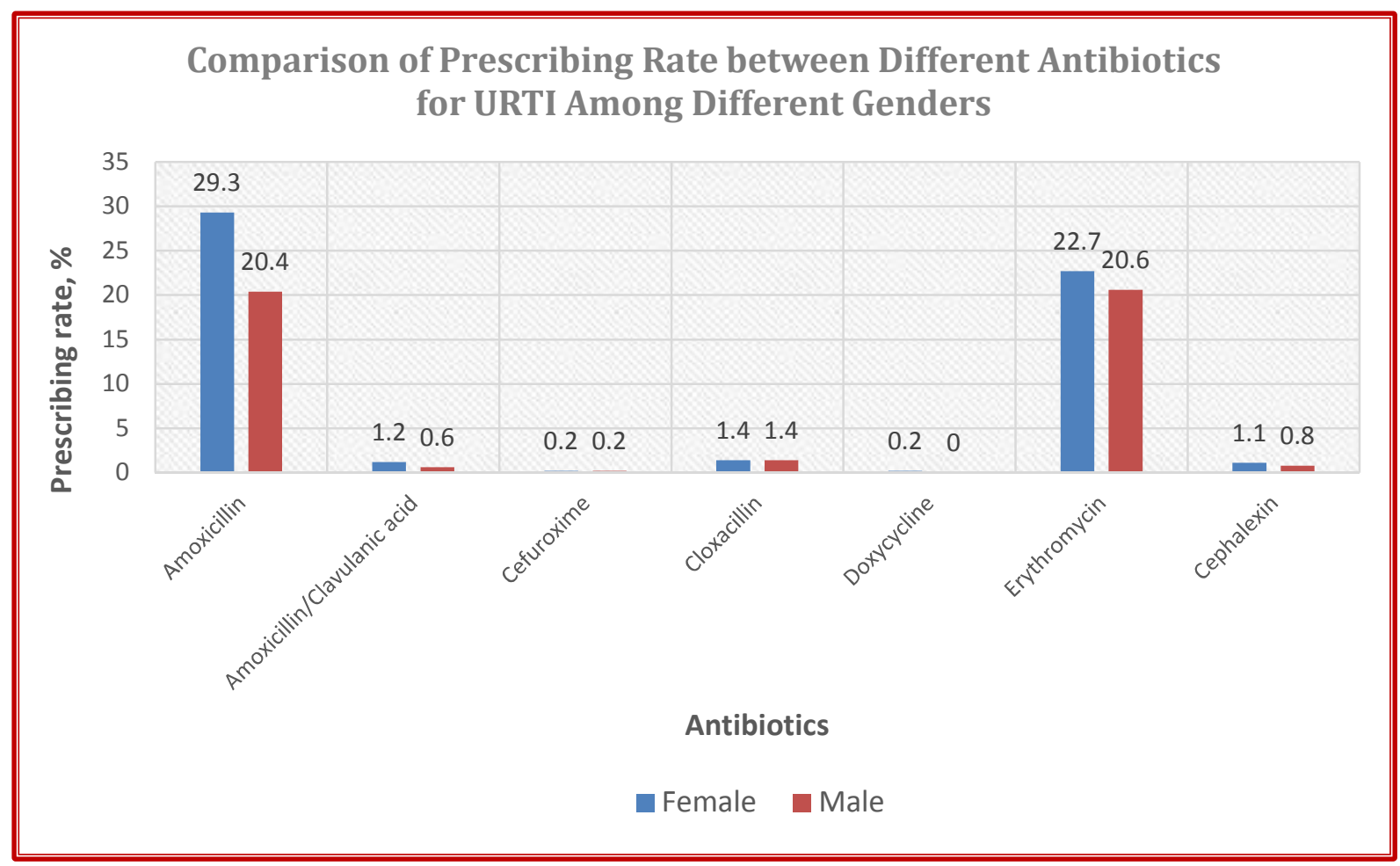

Figure 3: Comparison of antibiotic prescribing rate among different genders

\section{Factors affecting prescribing pattern according to ethnicity}

Figure 4 shows that Malays achieved the highest prescription rate for all the antibiotics. This is in congruence with the findings from Table 1 which indicates that Malay population was the highest (63.6\%) among all ethnicities. However, results show that the prescription rate of amoxicillin/clavulanic acid and cephalexin for Chinese were higher $(0.3 \%$ and $0.6 \%$ ) than Indians (0\% and $0.2 \%$ ) (Figure 4 ). This contradicts the data from Table 1 which indicates a higher population of Indians (22.3\%) compared to Chinese population (11.4\%). Nevertheless, statistical differences were observed in specific ethnicities. There was a statistically significant difference between other ethnicities with Malays $(p<0.001)$, Indians $(p<0.001)$ and Chinese $(p<0.05)$ for all the antibiotics indicated for URTI. Malays were found to have a statistically significant difference from Indians $(p<0.05)$ and Chinese $(p<0.001)$ for all the antibiotics indicated for URTI. 


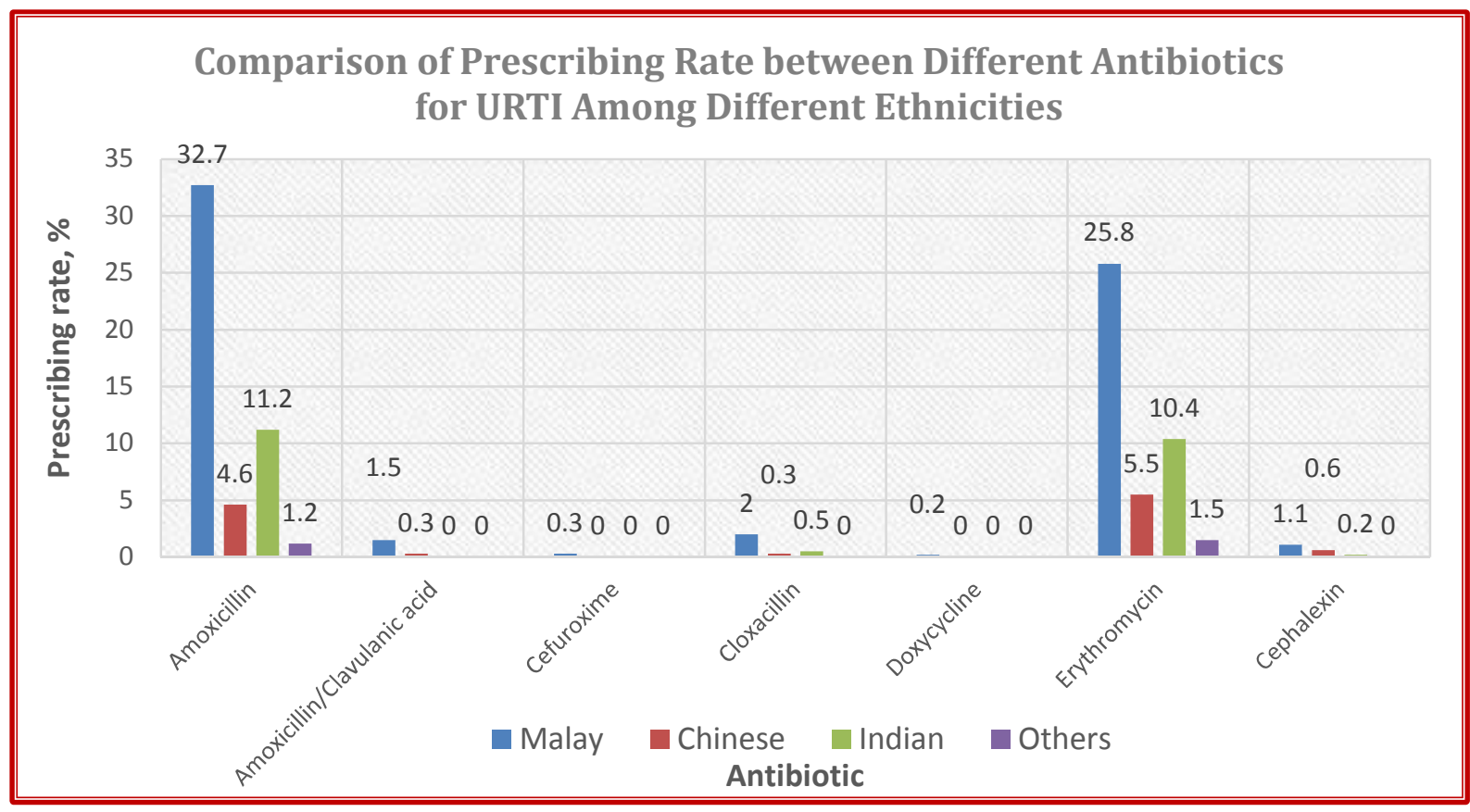

Figure 4: Comparison of antibiotics prescribing rate among different ethnicities

\section{Factors affecting prescribing pattern according to age group}

Patients were categorized into four groups according to their age; 18-29, 30-39, 40-49 and 50-60 years. Overall, patients aged 18-29 years were shown to have the highest prescribing rate for amoxicillin, erythromycin and cephalexin; patients aged 30-39 years were shown to have the highest prescribing rate for amoxicillin/clavulanic acid, cefuroxime, cloxacillin and doxycycline (Figure 5). The antibiotic prescribing rate in the age group of 18-29 years and 30-39 years were indicated to be about two times higher than patients in the age group of $50-60$ years (Table 1). Nevertheless, no significant differences were found in the prescribing pattern for all the antibiotics $(p>0.05)$.

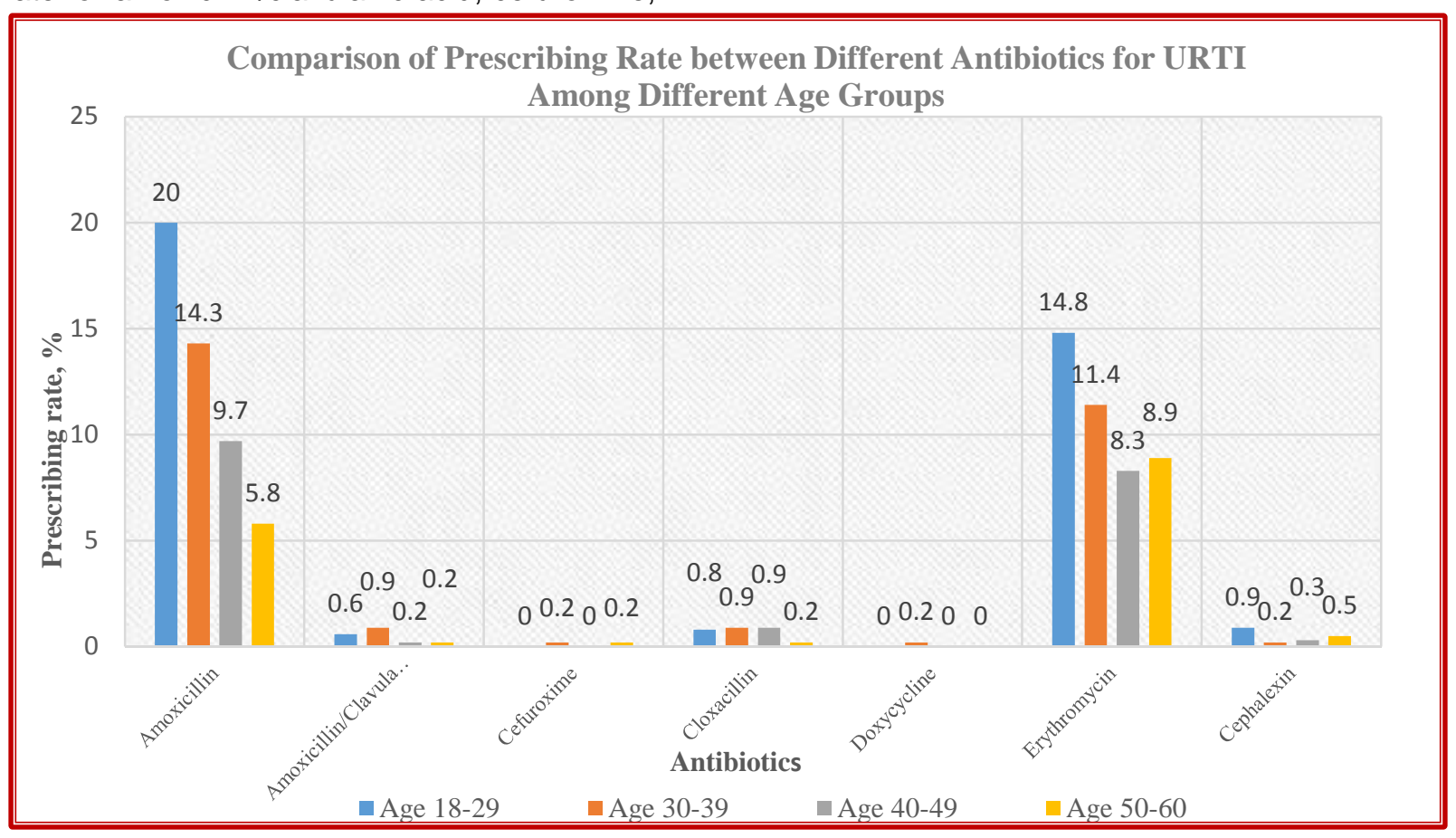

Figure 5: Comparison of antibiotics prescribing rate among different age groups 


\section{DDD of antibiotics}

The total dose prescribed was calculated to obtain the DDD/100 patients of each antibiotic for the indication of URTI in 2018 (Table 2). The most utilized antibiotics for URTI was amoxicillin (1.31 DDD/100 patients), and was used almost three-fold more than erythromycin which was the second most utilized antibiotic for URTI (0.48 DDD/100 patients). Other relatively less utilized antibiotics for URTI were cloxacillin (0.07 DDD/100 patients), amoxicillin/clavulanic acid (0.06 DDD/100 patients), cephalexin (0.02 DDD/100 patients), cefuroxime (0.01 DDD/100 patients) and doxycycline (0.01 DDD/100 patients). The DDD/100 patients for all antibiotics had a statistically significant association with its DDD. No statistical data could be generated for doxycycline as it consisted of only one sample data.

Table 2: ATC, DDD and DDD/100 patients of antibiotics (WHO 2018)

\begin{tabular}{|l|l|l|l|l|}
\hline Antibiotics & ATC code & DDD (gm) & $\begin{array}{l}\text { DDD/100 } \\
\text { patients (gm) }\end{array}$ & \\
\hline Amoxicillin & J01CA04 & 1.5 & 1.31 & $p<0.001$ \\
\hline Amoxicillin/Clavulanic Acid & J01CR02 & 1.5 & 0.06 & $p<0.001$ \\
\hline Cefuroxime & J01DC02 & 0.5 & 0.01 & $p<0.005$ \\
\hline Cloxacillin & J01CF02 & 2 & 0.07 & $p<0.001$ \\
\hline Doxycycline & J01AA02 & 0.1 & 0.01 & - \\
\hline Erythromycin & J01FA01 & 2 & 0.48 & $p<0.001$ \\
\hline Cephalexin & J01DB01 & 2 & 0.02 & $p<0.001$ \\
\hline
\end{tabular}

\section{Discussion}

The overusage of antibiotics is a global concern. Prescribing pattern needs to be revised from time to time to optimize medical care, rationalize prescribing practice and improve cost effectiveness. The current study was designed to evaluate the antibiotic prescribing pattern for URTI guided by MNAG and DDD system introduced by WHO. Study data was collected at Seremban 2 Health Clinic from 1st January 2018 to 31 st December 2018 and a study population of 651 patients aged between 18 to 60 years old were included in this study.

The overall antibiotic prescribing rate and URTI antibiotic prescribing rate in the current study were $11.6 \%$ and $25.2 \%$ respectively. The antibiotic prescribing rate for URTI was found to be similar to a few studies conducted in other public health clinics in Malaysia. For example the study conducted at Seremban Health Clinic in 2002 (Teng et al. 2004) and public healthcare centres in Kedah in 2014 (Rabiatul et al. 2015) showed an antibiotic prescribing rate of $26 \%$ and $32 \%$ respectively. In addition, studies done in public care clinics in 2008 and public health clinics in Perlis in 2010 and 2011 showed an antibiotic prescribing rate of $27.8 \%, 33.5 \%$ and $23.3 \%$ respectively (Cheong et al. 2011, Kamarudin and Noh 2013). In terms of international comparison, the antibiotic prescribing rate for URTI in this study is much lower compared to China's primary healthcare facilities (53\%) from 2009 to 2011 (Li et al. 2012). However, when compared to more developed countries such as the Netherlands (17\%) and Hong Kong $(5 \%)$ in 2010 , the antibiotic prescribing rate for URTI is still a cause of concern at our institution (Van den Broek et al. 2014, Kung et al. 2014). Nevertheless, the prescribing rates from these studies cannot be compared directly due to varying study settings, varying definition of URTI, study period, sample size and availability of antibiotics.

Table 3: Antibiotic recommendation in NAG 2014

\begin{tabular}{|l|l|l|}
\hline Clinical Problem & \multicolumn{1}{|c|}{ Preferred Choice } & \multicolumn{1}{|c|}{ Alternative Choice } \\
\hline Throat And Upper Respiratory & Phenoxymethylpenicillin & Amoxicillin 500mg PO \\
(Tonsillitis/Pharyngitis) & $500 \mathrm{mg}$ PO q12h for 10 days & q8-12h for 10 days \\
& or & Penicillin Allergy: \\
& Benzathine Penicillin & or \\
& $1.2 \mathrm{MU}$ IM, 1 single dose & Clindamycin 300-450mg PO q8h for 10 days \\
\hline
\end{tabular}


URTI is predominantly viral in origin and thus are relatively self-limiting. However, globally there are about $70-80 \%$ of patients with URTI being prescribed with antibiotics which varies with the causative organism. Therefore, there is a need to differentiate between viral and bacterial infections before prescribing any antibiotic treatment (Almeman et al. 2014). It is generally believed that bacteria which requires antibiotic treatment for URTI is Streptococcus pyogenes (Group A Streptococcus) (Bisno A L 2001). The modified Centor score as described by Mclsaac et al. (1998) has been introduced in the guideline to decide which patients do not require testing or antibiotic therapy, require throat culture/rapid antigen detection testing or empiric antibiotic therapy to reduce excessive use of antibiotics in patients with sore throats. Among the criteria to diagnose URTI include the absence of cough, age less than 14 years, fever, cervical adenopathy and presence of tonsillar exudates (NAG 2014, CDCP 2017). No antibiotics should be given to patients who have only one or none of the above features during clinical examination (CDCP 2017).

Referring to Table 3, MNAG recommended phenoxymethylpenicillin as the preferred drug and amoxicillin as the alternative drug choice for URTI. Thus, about half of the cases were prescribed with the appropriate antibiotic except for the usage of erythromycin, cloxacillin, doxycycline, cefuroxime and cephalexin. Amoxicillin was the most popular choice with a prescribing rate of $49.8 \%$. This is in line with the findings in the National Medical Care Statistics (NMCS) 2010 study on morbidity and prescription pattern in the public and private primary care settings, where amoxicillin was the most prescribed antibiotics for URTI at a rate of $35.0 \%$ (Arnold and Straus 2005). On the other hand, erythromycin which has been removed from the National Antibiotic Guideline since 2014 as a preferred treatment in URTIs due to a high resistance trend came up as the second most popular choice with a prescribing rate of $43.3 \%$. This may be due to ignorance from physicians or lack of information regarding the changes to MNAG. Besides that, the use of broad-spectrum antibiotics are not necessary as it is more likely to promote antibiotic resistance based on a study in Japan (Teng CL 2014). Although phenoxymethylpenicillin is available at the clinic and can be prescribed by medical officers, it was never used for URTI despite being the preferred choice of treatment. This may be due to the ease of its alternative regimen and cost effectiveness of amoxicillin compared to phenoxymethylpenicillin. Phenoxymethypenicillin is available as $125 \mathrm{mg}$ tablet with a unit price of RM0.47 whereas amoxicillin is available as $250 \mathrm{mg}$ capsule with a unit price of RM0.47. Thus, for the treatment, a total dose of 8 tablets of phenoxymethypenicillin 125mg (RM3.76) are needed compared to only 6 capsules of amoxicillin $250 \mathrm{mg}$ (RM2.82). This shows that amoxicillin is more cost effective compared to phenoxymethylpenicillin. However, the results from this study show that the current practice does not follow the antibiotic guidelines.

Referring to Table 1, 37\% of patients who were prescribed antibiotics for URTI were aged between 18 to 29 years old, followed by $27.9 \%$ aged between 30 to 39 years old, $19.4 \%$ aged between 40 to 49 years old and $15.7 \%$ aged between 50 to 60 years old. This shows that there was a higher incidence of URTI among younger age groups. This finding is comparable with findings of another study done in Malaysia (Rahman et al. 2016) where patients aged 20 to 39 years old were mostly prescribed with antibiotics. Malays contributed to more than half of the study population $(63.6 \%)$. This is in congruence with the huge number of Malay patients who visited the pharmacy in 2018 which was almost $60 \%$ of the total number of patients. Patient's gender was not shown to have a statistically significant association with the antibiotic prescribing pattern for URTI.

The Malaysian Statistics on Medicines (MSOM) reported that the three most utilized antibiotics were amoxicillin (DDD $0.73 \mathrm{~g}$ ), cephalexin (DDD $0.058 \mathrm{~g}$ ), and erythromycin (DDD $0.53 \mathrm{~g}$ ) (Faridah et al. 2019). This is in agreement with the top two utilized antibiotics in the current study which were amoxicillin (DDD/100 patients $1.31 \mathrm{~g}$ ) and erythromycin (DDD/100 patients 0.48). Nevertheless, the data collected from MSOM was a combination from private and government sectors (Faridah et al. 2019). Thus direct comparison cannot be made. Majority of the antibiotics did not comply with the DDD set by WHO (Table 2). This may be due to the different prescribing pattern and the fact that the DDD in the current study is solely based on the diagnosis for URTI, thus the dose may differ.

Biomedical factors which include clinical presentation of patients, characteristics of disease, availability of medicine as well as prescribers' knowledge can affect decision making. Nevertheless, other factors such as patients' and prescribers' attitude can also 
lead to different prescribing decisions. Moreover, as primary health clinics are often crowded with long waiting times, prescribers may prescribe antibiotics instead of taking time to explain why they are not needed. Antibiotics are often viewed by patients as a 'magic bullet' for all infections and prescribers are lacking in confidence to explain that URTI is self-limiting. Therefore, these issues need to be addressed. These include providing education and up to date information to prescribers regarding implementation of antibiotic guidelines and antimicrobial stewardship program. It is important to emphasize the use of penicillin as the first line of treatment for URTIs and remind the prescribers about the removal of erythromycin as an alternative treatment for URTIs due to increasing resistance. An antibiotic audit should be routinely carried out and the findings be discussed at drug committee meetings to optimize the utilisation of antibiotics. Thus, DDD is an important tool to gather information on antibiotic susceptibility patterns. It has been reported that with interventions, such as feedback of prescribing data to prescribers and academic detailing by senior family medicine specialist, a more prudent antibiotic use can be promoted. (Kamarudin and Noh 2013, Teng et al. 2006). On the other hand, patients should be educated regarding the unnecessary need of antibiotics in most of the URTI cases and regarding the emergence of antibiotic resistance. Public health education to fight against excessive use of antibiotics has been carried out in Malaysia and this effort needs to be persistent (Star 2012, 2015). This is supported by reviews from high-income countries which recommend public health campaign to encourage judicious antibiotic use. (Huttner et al. 2010) It is hoped that with this continuing effort to reach out to the public, awareness of the emergence of antibiotic resistance can be implemented. The rate and prescribing patterns of antibiotics can also be affected by the availability of diagnostic facilities. Diagnostic tests such as rapid screen test have demonstrated good performance at

\section{References}

Ab R.N., Teng C.L., Sivasampu S. (2016). Antibiotic prescribing in public and private practice: a cross-sectional study in primary care clinics in Malaysia. BMC Infectious Diseases.16(1), 208.

Akter S.F.U., Rani M.F.A., Rahman J.A., et al.(2012). Antimicrobial use and factors identifying streptococcal pharyngo-tonsillitis. However, due to its cost it is not a common tool to be used in Asia-Pacific countries (RuizAragón 2010). Hopefully in the near future, potentially greater diagnostic testing could be carried out at primary health clinics regularly as testing kits become inexpensive.

\section{Conclusion}

This study examined the antibiotic prescribing pattern and appropriateness of antibiotics prescribed for URTI in Seremban 2 Health Clinic. The most utilized antibiotics for URTI was found to be amoxicillin, followed by erythromycin as the second most prescribed antibiotic for URTI. MNAG have stated that phenoxymethylpenicillin is the first-line option for URTIs and erythromycin has long been removed from the guideline as a treatment option due to a high resistance trend. These results show that the current practice is not following the antibiotic guidelines. Factors contributing to non-adherence of guidelines should be investigated and actions should be taken to optimize the antibiotic prescribing trend. Thus, the collaboration between pharmacists and prescribers play an important role in monitoring the prescribing pattern of antibiotics.

\section{Acknowledgement:}

The authors would like to thank the Director General of Health Malaysia for his permission to publish this article.

\section{Ethical approval}

Ethical approval for this study was obtained from the Medical Research and Ethics Committee (MREC), Ministry of Health Malaysia.

\section{Conflict of interests}

There was no conflict of interest to disclose and no funding was received for this study.

influencing prescribing in medical wards of a tertiary care hospital in Malaysia. Int $J$ Sci Environ Technol. 1(4), 274-284.

Almeman A.A., Ibrahim M.I.M., Rasool S. (2014). Cost analysis of medications used in upper respiratory tract infections and prescribing patterns in university sans 
Malaysia, Penang, Malaysia. Tropical Journal of Pharmaceutical Research. 13(4), 621-626.

Arnold S.R., Straus S.E.(2005). Interventions to improve antibiotic prescribing practices in ambulatory care. Cochrane Database Syst Rev. 2005;4:CD003539

Bisno A.L. (2001). Acute pharyngitis. N Engl J Med. 344(3), 205-11.

Boonstra E., Lindbaek M., Ngome E. (2005). Adherence to management guidelines in acute respiratory infections and diarrhoea in children under 6 years old in primary health care in Botswana. Int J Qual Health Care;17(3), 221227.

Centers for Disease Control Prevention. Get smart for healthcare (2017). Retrieved 15 June 2019. Available from: www.cdc.gov/getsmart/healthcare

Cheong L.T., Seng F.T., Ee M.K., et al. Antibiotics for URTI and UTI Prescribing in Malaysian primary care settings. Australian Family Physician May 2011; 40:5.

Costelloe C., Metcalfe C., Lovering A., et al.(2010). Effect of antibiotic prescribing in primary care on antimicrobial resistance in individual patients: Systematic review and meta-analysis. BMJ,340,c2096.

Don't ask for antibiotics if not needed. In: The Star. Malaysia; 31 May 2015. Retrieved 16 June 2019. Available from: https://www.thestar.com.my/news/nation/2015/ 05/31/dont-ask-for-antibiotics-if-not-neededdoc-tough-to-convince-such-patients/

Faridah A.M., Sivasampu S., Lian L., et al. (2010) Malaysian Statistics on Medicine 2007. Retrieved 17 June 2019. Available from: https://vdocuments.site/malaysian-statisticson-medicines-2007291010.html

Fikru W. and Dagmawit T.. (2018). Retrospective Assessment of Antibiotics Prescribing at Public Primary Healthcare Facilities in Addis Ababa, Ethiopia. Hindawi Interdisciplinary Perspectives on Infectious Diseases,2018, 1-9. https://doi.org/10.1155/2018/4323769

Huttner B., Goossens H., Verheij T., Harbarth S. (2010). Characteristics and outcomes of public campaigns aimed at improving the use of antibiotics in outpatients in high-income countries. Lancet Infect Dis. 10(1),17-31.

Kamarudin Mohd. F. and Noh Kamaliah M. (2013). The effect of profiling report on antibiotic prescription for upper respiratory tract infection. Malays Fam Physician. 8(2), 26-31.

Kung K., Wong C.K., Wong S.Y., Lam A., Chan C.K., Griffiths S., Butler C. (2014). Patient presentation and physician management of upper respiratory tract infections: a retrospective review of over 5 million primary clinic consultations in Hong Kong. BMC Fam Pract. 15, 95.

Li Y., Xu J., Wang F. et al. (2012). Overprescribing in China, driven by financial incentives, results in very high use of antibiotics, injections, and corticosteroids. Health Affairs.31(5), 1075-82.

Mclsaac W.J., White D., Tannenbaum D., Low D.E. (1998). A clinical score to reduce unnecessary antibiotic use in patients with sore throat. Canadian Medical Association Journal. 158(1), 75-83.

Ministry of Health Malaysia (2018) Official portal. Retrieved 10 December 2018. Available from:

http://www.moh.gov.my/english.php/database stores/store view/1

O'Neill J. (2016). Tackling drug-resistant infections globally: Final report and recommendations. The review on antimicrobial resistance. Available from: https://amrreview.org/sites/default/files/160518 Final\%20 paper with\%20cover.pdf

Pharmaceutical Services Division. National Antibiotic Guideline (NAG), 2nd Edition. 2014. Retrieved 5 June 2019. Available from: http://www.pharmacy.gov.my/v2/ms/dokumen/ national-antibiotic-guideline-nag-

2ndedition.html.

Philips H., Huibers L., Holm Hansen E., et al. (2014). Guidelines adherence to lower urinary tract infection treatment in out-of-hours primary care in European countries. Qual Prim Care 22(4), 221-231.

Rabiatul S., Hassali M., Alrasheedy A.A.,et al. (2015). Prescribing Patterns for Upper 
Respiratory Tract Infections: A PrescriptionReview of Primary Care Practice in Kedah, Malaysia and the implications. Expert Rev Anti Infect Ther. 13(12),1547-56.

Rezel R.S., Hassali M.A., Alrasheedy A.A., et al. (2015). Prescribing patterns for upper respiratory tract infections: A prescription review of primary care practice in Kedah, Malaysia, and the implications. Expert Rev Anti Infect Ther, 13(12),1547-1556.

Rico-Ferreira P., Palazon-Bru A., Calvo-Perez M., Gil-Guillen V.F. (2015). Nonadherence to guidelines for prescribing antibiotic therapy to patients with tonsillitis or pharyngotonsillitis: A cross sectional study. Curr Med Res Opin., 31(7),1319-1322.

Ruiz-Aragón J., Rodríguez López R., Molina Linde J.M. (2010). Evaluation of rapid methods for detecting Streptococcus pyogenes. Systematic review and meta-analysis. An Pediatr (Barc). 72(6), 391-402

Shafinaz S., Muhammad E.A., Syed Tabish R.Z. et al. (2016). Antimicrobial drug use in primary healthcare clinics: a retrospective evaluation. International Journal of Infectious Diseases, 52, 16-22.

'Superbugs' spur campaign for stricter use of antibiotics. The Star. Malaysia; 29 September 2012. Retrieved 16 June 2019. Available from: http://www.thestar.com.my/news/nation/2012/0 9/29/superbugs-spur-campaign-for-stricteruse-of-antibiotics/

Teng C.L., Achike F.I., Phua K.L., et al. (2004). General and URTI-specific antibiotic prescription rates in a Malaysian primary care setting. International Journal of Antimicrobial Agents. 24(5), 496-501.

Teng C.L., Achike F.I., Phua K.L., Nurjahan M.I., Mastura I., Asiah H.N., Mariam A.M., Narayanan S., Norsiah A., Sabariah I., et al.(2006). Modifying antibiotic prescribing: the effectiveness of academic detailing plus information leaflet in a Malaysian primary care setting. Med J Malaysia. 61(3),323-31.

Teng C.L., Nurjahan M.I., Hashim N.A., et al.(2003). Upper respiratory tract infections: to what extent is the management evidencebased? Med J Malaysia, 58,159-66.
Teng C.L. (2014). Antibiotic prescribing for upper respiratory tract infection in the AsiaPacific region: a brief review. Malays Fam Physician,9(2), 18-25.

The World Health Organization. Antimicrobial resistance, global report on surveillance (2014) Retrieved 30 November 2018. Available from: http://apps.who.int/iris/bitstream/handle/10665/ 112642/9789241564748 eng.pdf;jsessionid $=4$ 788A03552E40DCBCED39A2F0D820491?se quence $=1$

Van den Broek d'Obrenan J., Verheij T.J, Numans M.E., van der Velden A.W. (2014). Antibiotic use in Dutch primary care: relation between diagnosis, consultation and treatment. $J$ Antimicrob Chemother. 69(6),1701-7.

WHO (2018) ATC/DDD Index 2019. Retrieved 4 December 2018. Available from: https://www.whocc.no/atc ddd index/

WHO (2018) Defined Daily Dose (DDD). Retrieved 4 December 2018. Available from: https://www.who.int/medicines/regulation/medi cines-safety/toolkit ddd/en/

WHO Collaborating Centre for Drug Statistics Methodology (2018). ATC/DDD Index 2019. Retrieved 5 June 2019. Available from: https://www.whocc.no/atc ddd index/

World Population Review (2018) Population of Cities in Malaysia (2018). Retrieved 10 November 2018. Available from: http://worldpopulationreview.com/countries/ma laysia-population/cities/

Zeyad A., Mohamed A., Abdullah A., et al. (2017). Study of antibiotics prescribing pattern in infants: Retrospective study: The study of drug utilization pattern in paediatric patients. International Journal of Medicine Research, 2(6), 16-22. 